\title{
Short-term effects of biochar and cake fertilizer on soil properties, plant growth, and fruit quality of Nanfeng tangerine
}

\author{
Qingjiang Wei ${ }^{1}$, Shouting $\mathrm{Su}^{1}$, Si Le ${ }^{1}$, Qiaoli $\mathrm{Ma}^{1}$, Xiao $\mathrm{Liu}^{2}$ and Qingqing $\mathrm{Gu}^{1}$ \\ ${ }^{1}$ College of Agronomy, Jiangxi Agricultural University, Nanchang, Jiangxi, China \\ ${ }^{2}$ School of Horticulture and Plant Protection, Yangzhou University, Yangzhou, Jiangsu, China
}

\section{Summary}

The acidic soil induces detrimental influences on citrus growth and development, which could be ameliorated using organic amendments, such as biochar and organic fertilizer. However, beneficial effects of biochar on fruit-bearing citrus orchards remain unknown. Here, we conducted a field experiment to compare the effects of biochar (B), cake fertilizer (CF), and their combined $(B+C F)$ application on physicochemical attributes of soil, growth performance and fruit quality of Nanfeng tangerine during a one-year sampling period. Results revealed that soil $\mathrm{pH}$, organic matter content, and available $\mathbf{N}$ and $K$ concentrations were increased by $B, C F$, and $B+C F$ applications. These applications also caused significant changes in microbial counts. Moreover, plant growth was largely improved by application of $B$ and $C F$ alone, and particularly by $B+C F$ combination. This was further supported by the observation that the three applications increased leaf nutrient concentrations of Nanfeng tangerine. Additionally, fruit size and mastication characteristic were improved by $B, C F$, and $B+C F$ applications. Increases in soluble sugars (fructose and glucose) and decline in citric acid content were observed in $\mathrm{CF}$ and $\mathrm{B}+\mathrm{CF}$ treated fruits. Vitamin $\mathrm{C}$ content increased, and the highest value was recorded for the CF applied treatments. Overall, this investigation shows the potential and role of biochar with cake fertilizer in enhancing the growth and fruit quality of Nanfeng tangerine as well as an improvement of soil properties. This is a preliminary investigation of the phenomenon, and further studies may focus on field applications in long-term experiments.

Keywords

biochar, cake fertilizer, fruit quality, Nanfeng tangerine, soil fertility

\section{Introduction}

Biochar is a carbon-rich solid material obtained by slow pyrolysis of biomasses in an oxygen-limited environment. Numerous studies have demonstrated that biochar can be used as a soil amendment to improve physicochemical properties of the soils (Schulz and Glaser, 2012; Al-Wabel et al., 2018; Chen et al., 2019). Biochar can influence soil aggregate and stability by interacting with soil organic matter, microorganisms, and minerals (Joseph et al., 2020). The biochar

\section{Significance of this study}

What is already known on this subject?

- Biochar improves soil properties and promotes crop growth and yield.

\section{What are the new findings?}

- Rice husk biochar with cake fertilizer enhanced the growth performance and fruit quality of Nanfeng tangerine as well as improved the acidic soil properties.

What is the expected impact on horticulture?

- Biochar in combination with organic fertilizers is beneficial for citrus plants, and could be widely used in citrus orchards, especially in the regions suffering from acidic soils.

application was beneficial for increasing soil organic carbon, cation exchange capacity (CEC), and available potassium, while it reduces soil bulk density (Abrishamkesh et al., 2015; Khan et al., 2019b). Furthermore, the high porosity in biochar contributes to improve soil water holding capacity (Pratiwi and Shinogi, 2016; Fischer et al., 2019). Positive effects on soil fertility are mainly explained by an increased $\mathrm{pH}$ in acidic soils (Van Zwieten et al., 2010; Wang and Liu, 2017). This improves nutrient retention via cation adsorption due to its high surface area and CEC (Liang et al., 2006), and benefits soil microorganisms such as mycorrhizal fungi and bacteria (Warnock et al., 2007).

For the positive effects on soil properties, biochar was widely used to promote crop growth and yield. For instance, application of biochar at $15 \mathrm{t} \mathrm{ha}^{-1}$ and $20 \mathrm{t} \mathrm{ha}^{-1}$ rates to the sandy soil increased the maize grain yield by $150 \%$ and $98 \%$, respectively (Uzoma et al., 2011). In tomato, Abid et al. (2017) found that biochar application enhanced plant shoot and root dry weight under cadmium contaminated condition. Another investigation also evidenced that biochar amendments when applied to the soil have increased plant height, fresh and dry weight of above and below ground biomass of tomato seedling (Khan et al., 2019b). Similar results have been observed in other crops such as sorghum, rice, quinoa, and onion (Steiner et al., 2007; Liu et al., 2016; Ramzani et al., 2017; Khan et al., 2019a). However, the effect of biochar on crop yield is related to time. Major et al. (2010) found that the maize yield was not increased in the first year of biochar application, while it was increased in the following three years. In recent years, the utility of biochar 
as a carrier for slow/controlled-release fertilizers has gained much importance (Chen et al., 2019). Studies found that biochar in combination with other fertilizers (such as inorganic fertilizer, farmyard manure, and green manure) could be a better strategy for crop yield improvement in comparison to sole biochar application (Partey et al., 2014; Arif et al., 2016; Nguyen et al., 2018; Singh et al., 2019).

Nanfeng tangerine (Citrus reticulata Blanco cv. 'Kinokuni') is a common citrus cultivar originated in Nanfeng County, Jiangxi Province, China. The cultivated area and yield of Nanfeng tangerine have increased in recent decades. However, most of the soil in the Nanfeng tangerine orchards is acidic, which influences the soil nutrient availability and causes the growth disorder, consequently could reduce the fruit quality, especially mastication characteristic (Zheng et al., 2015). Several studies have shown that the biochar increased the soil quality and the growth performance of citrus plants potted in acidic red soils (Zhang et al., 2015; Guo et al., 2016). However, no field study has been conducted to determine the effects of application of biochar on soil properties. Moreover, whether biochar could be used to improve fruit quality of Nanfeng tangerine is still unclear. Therefore, the present study aims to explore the interactive effects of biochar and cake fertilizer on the acidity, microbial and nutritional properties of the soil, and particularly to evaluate their beneficial effects on plant growth and fruit quality of Nanfeng tangerine.

\section{Materials and methods}

\section{Experimental site and set up}

The field experiment was conducted in a commercial orchard $\left(27^{\circ} 6^{\prime} 24^{\prime \prime} \mathrm{N}, 116^{\circ} 28^{\prime} 58^{\prime \prime} \mathrm{E}\right)$ located in Nanfeng County of Jiangxi Province, China. The soil was highly acidic red. Fifteen-year-old trees with $4 \mathrm{~m}$ spacing and similar growth vigor were selected as experimental materials. The experiment comprised three fertilization treatments with equal nitrogen input, including B (Rice husk biochar, $6.6 \mathrm{~kg} \mathrm{tree}^{-1}$ ), CF (cake fertilizer, $6 \mathrm{~kg}$ tree $^{-1}$ ), B+CF (3.3 kg biochar plus $3 \mathrm{~kg}$ cake fertilizer tre $\mathrm{e}^{-1}$ ), and no base fertilizer was set as control. Each fertilization treatment had 3 replications, with 5 trees for each replication. Each soil and fruit sample was pooled from at 5 individual plants. The fertilizers were applied in December 2016 by furrow application method and thoroughly plowing into the soil to a depth of $30 \mathrm{~cm}$. Rice husk biochar and oil cake organic fertilizer used in this study were commercially purchased from Huafeng Corporation, Ltd. (Jiangsu, China). During the study period, from December 2016 to December 2017, the cultural practices and plant protection measures of different treatments were consistent, and the plants were fertilized with farmer's fertilization practice. Basic properties of biochar and cake fertilizer are given in Table 1, which were determined according to standard methods as described by Zhang et al. (2015).

\section{Collection and analysis of soil samples}

Soil samples from each treatment were collected at two time points (December 2016 and December 2017), i.e., before and after treatments, respectively. The samples were collected from 0-30 cm depth to make composite samples and then shipped to the laboratory. After $2 \mathrm{~mm}$ sieving, a part of the soil samples was stored at $4^{\circ} \mathrm{C}$ for microbial count determination, and the other part was air-dried at room temperature to determine soil $\mathrm{pH}$, organic matter, and mineral concentrations.

Counts of soil bacteria, fungus, and actinomycete were determined by dilution method of plate counting (Li et al., 2008). Soil pH was determined potentiometrically in $1: 2.5$ soil/distilled water suspensions, using a pH meter (FE20K, Mettler Toledo, Zürich, Switzerland). Soil organic matter was determined by $\mathrm{K}_{2} \mathrm{Cr}_{2} \mathrm{O}_{7} / \mathrm{H}_{2} \mathrm{SO}_{4}$ oxidation. Soil was digested with $\mathrm{NaOH}$ and available $\mathrm{N}$ was measured using alkaline hydrolysis diffusion method. Available P was extracted with $\mathrm{NaHCO}_{3}$ and measured by a UV spectrophotometer (UV2600, Shimadzu, Kyoto, Japan). Available K was determined using an atomic absorption spectrophotometer (Z-2000, Hitachi, Tokyo, Japan) following digestion by $\mathrm{CH}_{3} \mathrm{COONH}_{4}$.

\section{Determination of plant growth and leaf nutrient concentration}

For plant growth analysis, 20 spring shoots were selected in July 2017, and their length and diameter were measured using the vernier caliper (Guanglu, Guilin, China). Meanwhile, 100 mature leaves were collected from non-fruiting spring branches of the treated trees. Then leaf area was determined by a portable leaf area meter (Top Instrument, Hangzhou, China). Leaf length, width, and thickness were measured manually using a vernier caliper, and leaf weight was measured using an electronic balance (LE203E, Mettler Toledo, Zürich, Switzerland). For nutrient determination, the leaves were oven-dried to constant mass and ground to a fine powder to pass a 40-mesh screen. Leaf total $\mathrm{N}$ was determined by auto discrete analyzer (Smartchem200, AMS Systea, Paris, France) after being digested with $\mathrm{H}_{2} \mathrm{SO}_{4} / \mathrm{H}_{2} \mathrm{O}_{2}$. For available $\mathrm{P}, \mathrm{K}, \mathrm{Ca}, \mathrm{Mg}, \mathrm{B}, \mathrm{Cu}, \mathrm{Zn}, \mathrm{Fe}$, and $\mathrm{Mn}$ concentrations, the dried powders were extracted with $\mathrm{HCl}$ and determined by inductively coupled plasma spectrometry (Iris Intrepid II, Thermo, Mass., U.S.A.).

\section{Determination of fruit quality}

Fruit samples were harvested at November 2017 (195 days after flowering), then stored in an icebox immediately and shipped to the laboratory. Fruit weight and size were measured using analytical balance and vernier caliper, respectively. Fruit color was measured using Chroma Meter CR-400 (CR-400, Minolta, Osaka, Japan). Citrus color index (CCI) was calculated as CCI $=1,000 \times a^{*} /\left(L^{*} \times b^{*}\right)$.

Indices of fruit texture quality including shear force, and fiber strength, ductility and tenacity of the segment was determined using TPA test of texture analyzer (TA.XT plus, SMS, England) according to the method of Wei et al. (2014). Total soluble solid content was determined using a PAL-1 refractometer (Atago, Tokyo, Japan). Contents of soluble sugar and titratable acid were determined by anthrone colorimetry

TABLE 1. Physiochemical properties of the experimental biochar and cake fertilizer.

\begin{tabular}{lcccccc}
\hline & $\mathrm{pH}$ & $\begin{array}{c}\text { Organic matter } \\
\left(\mathrm{g} \mathrm{kg}^{-1}\right)\end{array}$ & $\begin{array}{c}\text { Organic carbon } \\
\left(\mathrm{g} \mathrm{kg}^{-1}\right)\end{array}$ & $\begin{array}{c}\text { Total N } \\
\left(\mathrm{g} \mathrm{kg}^{-1}\right)\end{array}$ & $\begin{array}{c}\text { Total P } \\
\left(\mathrm{g} \mathrm{kg}^{-1}\right)\end{array}$ & $\begin{array}{c}\text { Total K } \\
\left(\mathrm{g} \mathrm{kg}^{-1}\right)\end{array}$ \\
\hline Rice husk biochar & 7.12 & 351 & 203 & 40.6 & 19.1 & 12.18 \\
Cake fertilizer & 5.45 & 726 & 421 & 44.3 & 18.6 & 13.14 \\
\hline
\end{tabular}


method and sodium hydroxide titration method, respectively. Vitamin C content was determined using 2,6-dichloroindophenol titration method (Gao, 2006). Contents of soluble sugar and organic acid components were determined by high-performance liquid chromatography (LC-20AT, Shimad$\mathrm{zu}$, Kyoto, Japan), using the methods described by Zhang et al. (2005).

\section{Statistical analysis}

All data were recorded and organized in Excel 2010 (Microsoft, Seattle, U.S.A.). The data were analyzed with SPSS 19.0 (SPSS Inc., Chicago, U.S.A.). The differences between means were subjected to analysis of variance (ANOVA) and separated following the Duncan's test at $\mathrm{P}<0.05$.

\section{Results}

\section{Characteristics of biochar and cake fertilizer}

Chemical analysis revealed that rice husk biochar had higher $\mathrm{pH}$ than cake fertilizer. However, the cake fertilizer had higher contents of organic matter and organic carbon than that of biochar. The cake fertilizer also had more abundant contents of $\mathrm{N}$ and $\mathrm{K}$, while it had less $\mathrm{P}$ than that of the biochar (Table 1).

\section{Effects of treatments on soil physiochemical properties}

In order to explore the effects of biochar on soil fertility, we analyzed some soil properties including total microbial counts, soil pH, organic matter content, and N, P, K concentra-

TABLE 2. Changes in microbial count, soil pH, organic matter content, and N, $\mathrm{P}, \mathrm{K}$ concentrations after biochar, cake fertilizer, and their combined application.

\begin{tabular}{|c|c|c|c|c|c|}
\hline & & Control & B & CF & $\mathrm{B}+\mathrm{CF}$ \\
\hline \multirow[t]{2}{*}{$\mathrm{pH}$} & Before treatment & $4.52 \pm 0.14 a$ & $4.38 \pm 0.06 a$ & $4.50 \pm 0.19 a$ & $4.46 \pm 0.15 a$ \\
\hline & After treatment & $4.12 \pm 0.09 b$ & $5.08 \pm 0.63 a$ & $4.56 \pm 0.47 a$ & $4.93 \pm 0.72 a$ \\
\hline \multirow[t]{3}{*}{ Organic matter $\left(\mathrm{g} \mathrm{kg}^{-1}\right)$} & Before treatment & $28.10 \pm 2.36 a$ & $31.33 \pm 1.32 a$ & $31.80 \pm 1.59 a$ & $29.40 \pm 0.40 \mathrm{a}$ \\
\hline & After treatment & $33.17 \pm 1.82 b$ & $60.17 \pm 4.56 \mathrm{a}$ & $44.87 \pm 4.15 b$ & $71.97 \pm 2.61 \mathrm{a}$ \\
\hline & Change (\%) & 18 & 92 & 41 & 145 \\
\hline \multirow[t]{3}{*}{$\mathrm{N}\left(\mathrm{mg} \mathrm{kg}^{-1}\right)$} & Before treatment & $131.33 \pm 4.04 a$ & $144.33 \pm 2.31 \mathrm{a}$ & $158.33 \pm 6.66 a$ & $140.67 \pm 8.50 a$ \\
\hline & After treatment & $142.00 \pm 5.29 \mathrm{c}$ & $247.67 \pm 18.15 b$ & $241.67 \pm 18.15 b$ & $357.00 \pm 14.93 a$ \\
\hline & Change (\%) & 8 & 72 & 53 & 154 \\
\hline \multirow[t]{3}{*}{$P\left(\mathrm{mg} \mathrm{kg}^{-1}\right)$} & Before treatment & $114.77 \pm 3.83 a$ & $115.30 \pm 5.94 a$ & $112.60 \pm 0.20 \mathrm{a}$ & $112.23 \pm 8.80 a$ \\
\hline & After treatment & $108.33 \pm 5.51 b$ & $127.33 \pm 5.13 a$ & $121.33 \pm 6.66 a b$ & $122.33 \pm 11.93 a b$ \\
\hline & Change (\%) & -6 & 10 & 8 & 9 \\
\hline \multirow[t]{3}{*}{$\mathrm{K}\left(\mathrm{mg} \mathrm{kg}^{-1}\right)$} & Before treatment & $249.00 \pm 12.77 a$ & $192.33 \pm 12.74 a$ & $220.33 \pm 14.05 a$ & $206.33 \pm 15.01 a$ \\
\hline & After treatment & $358.33 \pm 13.50 \mathrm{c}$ & $596.00 \pm 29.01 b$ & $597.00 \pm 35.03 b$ & $732.67 \pm 27.39 a$ \\
\hline & Change (\%) & 44 & 210 & 171 & 255 \\
\hline \multirow[t]{3}{*}{ Bacteria $\left(10^{-5} \mathrm{CFU} \mathrm{g}^{-1}\right)$} & Before treatment & $24.67 \pm 2.31 d$ & $50.67 \pm 4.16 c$ & $71.78 \pm 3.79 \mathrm{~b}$ & $141.33 \pm 8.50 \mathrm{a}$ \\
\hline & After treatment & $52.33 \pm 4.51 \mathrm{c}$ & $141.33 \pm 14.27 b$ & $129.00 \pm 1.00 \mathrm{~b}$ & $203.50 \pm 16.50 a$ \\
\hline & Change (\%) & 112 & 179 & 80 & 44 \\
\hline \multirow[t]{3}{*}{ Fungi $\left(10^{-2} \mathrm{CFU} \mathrm{g}^{-1}\right)$} & Before treatment & $109.75 \pm 5.17 a$ & $109.67 \pm 5.13 a$ & $108.67 \pm 2.31 \mathrm{a}$ & $118.67 \pm 6.51 \mathrm{a}$ \\
\hline & After treatment & $72.11 \pm 6.74 d$ & $221.33 \pm 18.58 b$ & $166.22 \pm 14.24 \mathrm{c}$ & $289.33 \pm 14.05 a$ \\
\hline & Change $(\%)$ & -34 & 102 & 53 & 144 \\
\hline \multirow[t]{3}{*}{ Actinomycetes $\left(10^{-3} \mathrm{CFU} \mathrm{g}^{-1}\right)$} & Before treatment & $88.44 \pm 4.53 b$ & $109.67 \pm 2.08 a$ & $96.00 \pm 1.73 b$ & $106.00 \pm 4.36 \mathrm{a}$ \\
\hline & After treatment & $26.33 \pm 2.52 d$ & $78.11 \pm 4.55 b$ & $48.67 \pm 5.33 c$ & $116.44 \pm 11.34 a$ \\
\hline & Change (\%) & -70 & -29 & -49 & 10 \\
\hline
\end{tabular}

Each value represents mean \pm standard errors of three replicates $(n=15)$. Different lowercase letters within a row indicate significant differences after treatments $(P<0.05)$. Change indicates the increase or decrease percentage of each treatment. B: $6.6 \mathrm{~kg}$ rice husk biochar per tree; $C F: 6 \mathrm{~kg}$ cake fertilizer per tree; $\mathrm{B}+\mathrm{CF}: 3.3 \mathrm{~kg}$ biochar plus $3 \mathrm{~kg}$ cake fertilizer per tree.

TABLE 3. Leaf and shoot growth parameters of Nanfeng tangerine for the different treatments.

\begin{tabular}{lrrrr}
\hline & Control & \multicolumn{1}{c}{ B } & \multicolumn{1}{c}{ CF } & B+CF \\
\hline Leaf area $\left(\mathrm{cm}^{2}\right)$ & $24.36 \pm 0.33 \mathrm{~d}$ & $26.67 \pm 0.58 \mathrm{c}$ & $28.52 \pm 0.34 \mathrm{~b}$ & $31.06 \pm 0.38 \mathrm{a}$ \\
Leaf length $(\mathrm{cm})$ & $8.43 \pm 0.12 \mathrm{~d}$ & $10.40 \pm 0.17 \mathrm{c}$ & $11.72 \pm 0.21 \mathrm{~b}$ & $13.27 \pm 0.12 \mathrm{a}$ \\
Leaf width $(\mathrm{cm})$ & $4.20 \pm 0.02 \mathrm{~d}$ & $4.37 \pm 0.02 \mathrm{c}$ & $4.53 \pm 0.04 \mathrm{~b}$ & $4.86 \pm 0.02 \mathrm{a}$ \\
Leaf thickness $(\mathrm{mm})$ & $29.53 \pm 0.40 \mathrm{~b}$ & $32.33 \pm 0.63 \mathrm{a}$ & $34.23 \pm 1.48 \mathrm{a}$ & $33.30 \pm 1.25 \mathrm{a}$ \\
Fresh weight $(\mathrm{g})$ & $0.29 \pm 0.01 \mathrm{c}$ & $0.32 \pm 0.00 \mathrm{~b}$ & $0.37 \pm 0.01 \mathrm{a}$ & $0.37 \pm 0.01 \mathrm{a}$ \\
Dry weight $(\mathrm{g})$ & $0.12 \pm 0.01 \mathrm{c}$ & $0.13 \pm 0.00 \mathrm{c}$ & $0.15 \pm 0.01 \mathrm{~b}$ & $0.16 \pm 0.01 \mathrm{a}$ \\
Shoot length $(\mathrm{mm})$ & $46.61 \pm 0.37 \mathrm{c}$ & $54.15 \pm 0.23 \mathrm{~b}$ & $54.07 \pm 0.89 \mathrm{~b}$ & $56.98 \pm 0.47 \mathrm{a}$ \\
Shoot diameter $(\mathrm{mm})$ & $1.54 \pm 0.01 \mathrm{c}$ & $1.57 \pm 0.01 \mathrm{c}$ & $1.79 \pm 0.01 \mathrm{~b}$ & $2.08 \pm 0.05 \mathrm{a}$ \\
\hline
\end{tabular}

Each value represents mean \pm standard errors of three replicates $(n=15)$. Different lowercase letters within a row indicate significant differences among treatments $(P<0.05)$. B: $6.6 \mathrm{~kg}$ rice husk biochar per tree; $C F: 6 \mathrm{~kg}$ cake fertilizer per tree; $B+C F: 3.3 \mathrm{~kg}$ biochar plus $3 \mathrm{~kg}$ cake fertilizer per tree. 
TABLE 4. Nutrient concentrations in leaves of Nanfeng tangerine for the different treatments.

\begin{tabular}{|c|c|c|c|c|}
\hline & Control & B & $\mathrm{CF}$ & $\mathrm{B}+\mathrm{CF}$ \\
\hline $\mathrm{N}\left(\mathrm{g} \mathrm{kg}^{-1}\right)$ & $35.17 \pm 1.12 b$ & $38.30 \pm 1.80 a b$ & $39.05 \pm 2.65 a$ & $38.75 \pm 0.35 a$ \\
\hline$P\left(g_{k g}^{-1}\right)$ & $0.94 \pm 0.02 b$ & $0.98 \pm 0.04 a b$ & $1.05 \pm 0.05 a$ & $1.00 \pm 0.04 a b$ \\
\hline $\mathrm{K}\left(\mathrm{g} \mathrm{kg}^{-1}\right)$ & $14.37 \pm 0.35 b$ & $14.77 \pm 0.74 a b$ & $15.55 \pm 0.55 a$ & $15.00 \pm 0.26 a b$ \\
\hline $\mathrm{Ca}\left(\mathrm{g} \mathrm{kg}^{-1}\right)$ & $10.00 \pm 0.82 b$ & $16.97 \pm 1.16 a$ & $17.10 \pm 0.30 \mathrm{a}$ & $17.47 \pm 0.55 a$ \\
\hline $\operatorname{Mg}\left(\mathrm{g} \mathrm{kg}^{-1}\right)$ & $1.13 \pm 0.02 b$ & $1.22 \pm 0.13 a b$ & $1.28 \pm 0.05 a$ & $1.29 \pm 0.02 a$ \\
\hline $\mathrm{B}\left(\mathrm{mg} \mathrm{kg}^{-1}\right)$ & $120.50 \pm 5.50 b$ & $126.00 \pm 6.56 \mathrm{ab}$ & $130.33 \pm 0.58 a$ & $123.00 \pm 2.00 \mathrm{ab}$ \\
\hline $\mathrm{Cu}\left(\mathrm{mg} \mathrm{kg}^{-1}\right)$ & $4.07 \pm 0.59 b$ & $5.84 \pm 1.02 \mathrm{ab}$ & $6.26 \pm 1.70 \mathrm{a}$ & $6.10 \pm 0.30 \mathrm{ab}$ \\
\hline $\mathrm{Zn}\left(\mathrm{mg} \mathrm{kg}^{-1}\right)$ & $91.17 \pm 8.75 b$ & $106.83 \pm 10.68 a b$ & $111.87 \pm 9.80 a$ & $110.90 \pm 3.92 a$ \\
\hline $\mathrm{Fe}\left(\mathrm{mg} \mathrm{kg}^{-1}\right)$ & $194.95 \pm 5.65 \mathrm{c}$ & $221.10 \pm 15.50 b$ & $311.27 \pm 76.79 a$ & $297.20 \pm 19.56 \mathrm{ab}$ \\
\hline $\operatorname{Mn}\left(\mathrm{mg} \mathrm{kg}^{-1}\right)$ & $362.87 \pm 16.22 b$ & $506.60 \pm 50.31 a$ & $500.65 \pm 38.15 a$ & $550.40 \pm 16.10 a$ \\
\hline
\end{tabular}

Each value represents mean \pm standard errors of three replicates $(n=15)$. Different lowercase letters within a row indicate significant differences among treatments $(P<0.05)$. B: $6.6 \mathrm{~kg}$ rice husk biochar per tree; $C F: 6 \mathrm{~kg}$ cake fertilizer per tree; $\mathrm{B}+\mathrm{CF}: 3.3 \mathrm{~kg}$ biochar plus $3 \mathrm{~kg}$ cake fertilizer per tree.

tions of the treated soils (Tables 2-3). The result showed that soil $\mathrm{pH}$ was declined under the control while was increased by different treatments. The $\mathrm{pH}$ values of three treatments were significantly higher than that of the control after one year of treatments (Table 2). In addition, organic matter contents were differentially increased by four treatments, with the largest increase under the B + CF treatment (from 29.40 to $71.97 \mathrm{~g} \mathrm{~kg}^{-1}$ ), followed by the $\mathrm{B}, \mathrm{CF}$, and control treatments. Soil available $\mathrm{N}$ and $\mathrm{K}$ concentrations were significantly increased by the treatments, with the largest increase under $\mathrm{B}+\mathrm{CF}$ treatment, followed by the $\mathrm{B}, \mathrm{CF}$, and control treatments. The soil available $P$ concentration was not influenced by all the treatments (Table 2).

Counts of bacterial colony were largely different among four soil samples before treatments. The highest value was recorded in the $\mathrm{B}+\mathrm{CF}$ soils, and the lowest value was recorded in the control soils. After treatments, the bacterial counts were significantly increased $179,112,80$, and $44 \%$ by the $\mathrm{B}$, control, $\mathrm{CF}$, and $\mathrm{B}+\mathrm{CF}$ treatment, respectively. Moreover, soil fungi counts showed no significant difference among four soil samples before treatments. However, the counts were increased from 109.67 to $221.3310^{-2} \mathrm{CFU} \mathrm{g}^{-1}, 108.67$ to $166.2210^{-2} \mathrm{CFU} \mathrm{g}^{-1}$, and 118.67 to $289.3310^{-2} \mathrm{CFU} \mathrm{g}^{-1}$ under the $\mathrm{B}, \mathrm{CF}$, and $\mathrm{B}+\mathrm{CF}$ treatments, respectively, while they were declined by the control treatment. As for the actinomycete counts, they were significantly decreased by control, B, and $\mathrm{CF}$ treatment, among which the control was affected drastically. However, B + CF treatment slightly increased the actinomycete counts (Table 2).

\section{Effects of treatments on plant growth and leaf nutrient concentrations of Nanfeng tangerine}

The leaf area, leaf length and width were all significantly affected by the treatments, with the largest increases under B $+\mathrm{CF}$, followed by CF and B application. Compared with the control, the leaf thickness was significantly increased by $\mathrm{B}, \mathrm{CF}$, and $\mathrm{B}+\mathrm{CF}$ applications, but there were no significant differences among the three applications. The leaf fresh weight was also significantly increased by the three applications, while the B treatment did not affect leaf dry weight, which was increased by CF and B + CF treatments. Similarly, both the shoot length and diameter were increased by the different treatments, particularly by the B + CF treatment, although the shoot diameter showed no significant differences between the $\mathrm{B}$ treatment and the control. Compared to the control, the B, CF, and B $+\mathrm{CF}$ application increased leaf nutrient concentrations. However, no significant difference in the nutrients except for Fe was observed among these three applications (Table 4).

\section{Effects of treatments on fruit quality of Nanfeng tangerine}

Treatments effects on various fruit traits of Nanfeng tangerine are shown in Table 5. Maximum fruit size including fruit weight, fruit transverse and longitudinal diameters were found in the $\mathrm{B}+\mathrm{CF}$ treatment followed by $\mathrm{CF}$ and $\mathrm{B}$ fertilizers. However, no significant changes in fruit shape index were observed among the different treatments. The $\mathrm{B}+\mathrm{CF}$ treatment had no effect on citrus color index, but it was decreased by the B or CF treatment (Table 5). Some texture indices were also determined to evaluate the fruit mastication characteristic. The results revealed that the segment shear force was significant different among the four treatments, with the highest value observed in the control and the lowest value in the CF treatment. The control fruits also had the highest values of fiber strength, fiber ductility, and fiber tenacity, while the lowest values were observed in the CF or $\mathrm{B}+\mathrm{CF}$-treated fruits (Table 5). Furthermore, contents of vitamin C, sugars and acids were also significantly affected by the treatments. The $\mathrm{CF}$ treated fruits had the highest vitamin $\mathrm{C}$ content, followed by the $\mathrm{B}, \mathrm{B}+\mathrm{CF}$, and control fruits. The $\mathrm{CF}$ treated fruits also had the highest contents of total soluble solid (15.20\%) and soluble sugar (2.18\%). The titratable acid contents were similar between the control and B treated fruits, which were significant higher than that in the CF and $\mathrm{B}+\mathrm{CF}$ treated fruits. Compared with the control, total soluble solid/titratable acid ratio was significantly increased by $\mathrm{B}$, $\mathrm{CF}$, and $\mathrm{B}+\mathrm{CF}$ applications, and the $\mathrm{B}+\mathrm{CF}$ treated fruits had highest value (80.74).

The main components of soluble sugar and organic acid in Nanfeng tangerine fruits were also determined. As shown in Table 6, the fructose content was the highest $\left(29.72 \mathrm{mg} \mathrm{g}^{-1}\right.$ $\mathrm{FW}$ ) in $\mathrm{B}+\mathrm{CF}$ treated fruits, followed by $\mathrm{CF}, \mathrm{B}$ and the control fruits, but no significant differences were observed among the latter three fruits. The glucose contents were similar between the $\mathrm{CF}$ and $\mathrm{B}+\mathrm{CF}$ treated fruits, which were significantly higher than that of the $B$ treated fruits and the control fruits. The $\mathrm{B}+\mathrm{CF}$ treated fruits also had the highest sucrose content (77.29 $\left.\mathrm{mg} \mathrm{g}^{-1} \mathrm{FW}\right)$, followed by CF and B treated fruits, and the control fruits had the lowest content (72.88 $\mathrm{mg} \mathrm{g}^{-1} \mathrm{FW}$ ). Furthermore, the citric acid content of $\mathrm{CF}$ treated fruits was similar to the $\mathrm{B}+\mathrm{CF}$, which was significantly higher than that of the B treated fruits but lower than the control fruits. However, no significant difference in malic 
TABLE 5. Fruit quality of Nanfeng tangerine for the different treatments.

\begin{tabular}{lcccc}
\hline & Control & B & CF & B+CF \\
\hline Single fruit weight $(\mathrm{g})$ & $27.83 \pm 0.16 \mathrm{c}$ & $33.14 \pm 0.99 \mathrm{~b}$ & $33.70 \pm 0.83 \mathrm{~b}$ & $35.54 \pm 1.28 \mathrm{a}$ \\
Transverse diameter $(\mathrm{mm})$ & $40.79 \pm 0.15 \mathrm{c}$ & $42.28 \pm 0.49 \mathrm{~b}$ & $42.42 \pm 0.63 \mathrm{ab}$ & $43.21 \pm 0.19 \mathrm{a}$ \\
Longitudinal diameter $(\mathrm{mm})$ & $30.67 \pm 0.14 \mathrm{c}$ & $31.29 \pm 0.07 \mathrm{bc}$ & $31.58 \pm 0.52 \mathrm{~b}$ & $32.33 \pm 0.45 \mathrm{a}$ \\
Fruit shape index & $0.75 \pm 0.01 \mathrm{a}$ & $0.74 \pm 0.00 \mathrm{a}$ & $0.75 \pm 0.00 \mathrm{a}$ & $0.75 \pm 0.01 \mathrm{a}$ \\
Citrus color index & $3.95 \pm 0.02 \mathrm{a}$ & $3.45 \pm 0.05 \mathrm{c}$ & $3.80 \pm 0.02 \mathrm{~b}$ & $3.91 \pm 0.02 \mathrm{a}$ \\
Shear force $(\mathrm{g})$ & $2,248.33 \pm 28.74 \mathrm{a}$ & $2,045.62 \pm 25.56 \mathrm{~b}$ & $1,728.11 \pm 11.40 \mathrm{c}$ & $1,763.99 \pm 27.33 \mathrm{c}$ \\
Fiber strength $(\mathrm{g})$ & $133.85 \pm 2.30 \mathrm{a}$ & $127.02 \pm 2.30 \mathrm{~b}$ & $108.52 \pm 2.23 \mathrm{c}$ & $124.29 \pm 2.36 \mathrm{~b}$ \\
Fiber ductility $\left(\mathrm{g} \mathrm{s}^{-1}\right)$ & $34.29 \pm 1.20 \mathrm{a}$ & $26.68 \pm 0.44 \mathrm{c}$ & $30.32 \pm 0.23 \mathrm{~b}$ & $25.42 \pm 0.29 \mathrm{~d}$ \\
Fiber tenacity $\left(\mathrm{g} \mathrm{s}^{-1}\right)$ & $140.20 \pm 0.37 \mathrm{a}$ & $128.54 \pm 0.17 \mathrm{~b}$ & $122.49 \pm 1.24 \mathrm{c}$ & $125.66 \pm 2.80 \mathrm{~b}$ \\
Vitamin C (mg 100 $\left.\mathrm{g}^{-1} \mathrm{FW}\right)$ & $25.44 \pm 1.62 \mathrm{~b}$ & $28.71 \pm 0.70 \mathrm{ab}$ & $30.58 \pm 1.62 \mathrm{a}$ & $26.61 \pm 2.43 \mathrm{~b}$ \\
Total soluble solid $(\%)$ & $13.44 \pm 0.48 \mathrm{c}$ & $14.86 \pm 0.05 \mathrm{ab}$ & $15.20 \pm 0.10 \mathrm{a}$ & $14.40 \pm 0.11 \mathrm{~b}$ \\
Soluble sugar $(\%)$ & $1.49 \pm 0.04 \mathrm{c}$ & $1.72 \pm 0.06 \mathrm{~b}$ & $2.18 \pm 0.07 \mathrm{a}$ & $2.06 \pm 0.14 \mathrm{a}$ \\
Titratable acid (\%) & $0.23 \pm 0.01 \mathrm{a}$ & $0.22 \pm 0.02 \mathrm{a}$ & $0.19 \pm 0.01 \mathrm{~b}$ & $0.18 \pm 0.01 \mathrm{~b}$ \\
Total soluble solid / Titratable acid & $59.31 \pm 1.58 \mathrm{c}$ & $68.03 \pm 5.19 \mathrm{~b}$ & $78.89 \pm 5.01 \mathrm{a}$ & $80.74 \pm 3.01 \mathrm{a}$ \\
\hline
\end{tabular}

Each value represents mean \pm standard errors of three replicates $(n=15)$. Different lowercase letters within a row indicate significant differences among treatments $(P<0.05)$. B: $6.6 \mathrm{~kg}$ rice husk biochar per tree; $C F: 6 \mathrm{~kg}$ cake fertilizer per tree; $\mathrm{B}+\mathrm{CF}: 3.3 \mathrm{~kg}$ biochar plus $3 \mathrm{~kg}$ cake fertilizer per tree.

TABLE 6. Contents of sugar and organic acid components of Nanfeng tangerine fruit for the different treatments.

\begin{tabular}{lcccc}
\hline & Control & B & CF & B+CF \\
\hline Fructose $\left(\mathrm{mg} \mathrm{g}^{-1} \mathrm{FW}\right)$ & $26.00 \pm 0.21 \mathrm{~b}$ & $26.61 \pm 0.26 \mathrm{~b}$ & $27.79 \pm 0.74 \mathrm{ab}$ & $29.72 \pm 1.97 \mathrm{a}$ \\
Glucose $\left(\mathrm{mg} \mathrm{g}^{-1} \mathrm{FW}\right)$ & $40.29 \pm 1.02 \mathrm{c}$ & $43.69 \pm 0.21 \mathrm{~b}$ & $45.70 \pm 0.77 \mathrm{a}$ & $45.67 \pm 0.92 \mathrm{a}$ \\
Sucrose $\left(\mathrm{mg} \mathrm{g}^{-1} \mathrm{FW}\right)$ & $72.88 \pm 0.92 \mathrm{c}$ & $74.62 \pm 0.53 \mathrm{~b}$ & $74.21 \pm 1.82 \mathrm{bc}$ & $77.29 \pm 1.58 \mathrm{a}$ \\
Citric acid $\left(\mathrm{mg} \mathrm{g}^{-1} \mathrm{FW}\right)$ & $8.11 \pm 0.40 \mathrm{a}$ & $6.90 \pm 0.16 \mathrm{c}$ & $7.44 \pm 0.10 \mathrm{~b}$ & $7.49 \pm 0.19 \mathrm{~b}$ \\
Malic acid $\left(\mathrm{mg} \mathrm{g}^{-1} \mathrm{FW}\right)$ & $2.66 \pm 0.12 \mathrm{a}$ & $2.38 \pm 0.07 \mathrm{a}$ & $2.60 \pm 0.41 \mathrm{a}$ & $2.57 \pm 0.12 \mathrm{a}$ \\
Quinic acid $\left(\mathrm{mg} \mathrm{g}^{-1} \mathrm{FW}\right)$ & $0.08 \pm 0.01 \mathrm{a}$ & $0.08 \pm 0.00 \mathrm{a}$ & $0.08 \pm 0.01 \mathrm{a}$ & $0.07 \pm 0.07 \mathrm{a}$ \\
\hline
\end{tabular}

Each value represents mean \pm standard errors of three replicates $(n=15)$. Different lowercase letters within a row indicate significant differences among treatments $(P<0.05)$. B: $6.6 \mathrm{~kg}$ rice husk biochar per tree; $C F: 6 \mathrm{~kg}$ cake fertilizer per tree; $\mathrm{B}+\mathrm{CF}: 3.3 \mathrm{~kg}$ biochar plus $3 \mathrm{~kg}$ cake fertilizer per tree.

acid and quinic acid contents were observed among all treatments.

\section{Discussion}

It has been reported that one third of the total area of citrus orchards in Jiangxi province, one of the main citrus-producing regions in China, suffers from acidic soil (Guo et al., 2016). Indeed, we found that soil pH of all tested samples were about 4.5 (Table 2), which is lower than the appropriate standard for citrus growth. The acidic soil $\mathrm{pH}$ was significantly increased by application of biochar or biochar with cake fertilizer, but not by sole cake fertilizer application. This could be attributed to the biochar produced from the pyrolysis of rice husk being alkaline in nature that neutralizes soil acidity directly. Our results are consistent with the finding that the biochar application significant increases the $\mathrm{pH}$ of acidic soil (Alburquerque et al., 2013; Zhang et al., 2015). Since the biochar and cake fertilizer contain significant amount of plant nutrients (Table 1), they also increased the concentrations of soil organic matter and available $\mathrm{N}$ and $\mathrm{K}$ (Table 2). However, soil available $\mathrm{P}$ was not affected by any treatments. The reason for this remains to be examined, but one possibility is that the soils of Nanfeng tangerine orchards are acidic and have a strong fixation effect of phosphorus. Furthermore, we found that the integration of biochar and cake fertilizer showed better result to improve the soil organic matter and available nutrient concentrations than the solely application of biochar or cake fertilizer. These results suggest that there are potential complementary and/or synergy effects between the biochar and cake fertilizer when they are simultaneously applied into the soils (Partey et al., 2014; Adekiya et al., 2019).

Due to the multi-porous structure and specific surface area, biochar can adsorb soluble organic matter, gasses, and mineral nutrients, and thereby it provides a suitable habitat for microbial growth and reproduction (Warnock et al., 2007; Sandhu et al., 2019). Zhang et al. (2015) reported that soil microbial biomass and activities were increased by peanut hull and rice straw biochar. Similarly, our results revealed that the abundance of soil bacteria, especially the fungus, were obviously effect by the biochar applications. Fungi are capable of decomposing complex carbon compounds, improving soil quality, and promoting plant growth in a symbiotic manner (Guo et al., 2016; Amendola et al., 2017). However, the soil actinomycetes were decreased after treatments except for the B $+\mathrm{CF}$ application. In a study conducted in a rice-wheat rotation system, Bai et al. (2019) found the straw-derived biochar increased fungi decomposing biosolids, but decreased pathogenic fungi and $\mathrm{CO}_{2}$-emission-related fungi. Thus, biochar can improve soil bacterial properties by stimulating the proliferation of beneficial microbes while suppressing the pathogen abundance (Wang et al., 2019). To fully ascertain the impacts of rice hush biochar addition on microbial change relating acidic soil quality, further research on soil microbial community abundance, composition and activity are needed. 
The current study revealed that leaf and shoot growth of Nanfeng tangerine was improved by three treatments, particularly by the $\mathrm{B}+\mathrm{CF}$ application. This is in line with earlier findings that the growth parameters, such as plant height, biomass, shoot diameter, and root length of trifoliate orange seedling, were increased significantly after biochar was added to soils (Zhang et al., 2015; Guo et al., 2016). In addition, maize shoot and grain yield were generally higher in mixed biochar with green manure than single treatment applications because of increased $\mathrm{N}$ use efficiency (Partey et al., 2014). We found that the response of leaf nutrients to the biochar, cake fertilizer, and their combined application was consistent with the values of soil properties recorded for these treatments. Biochar have the ability to improve soil interactive ability and nutrient utilization of inorganic and organic fertilizers by enhancing microbial functions and soil organic matter and reducing nutrient loss, thereby providing an ideal condition for improving nutrient synchrony with crop nutrient demands (Arif et al., 2016; Al-Wabel et al., 2018). We conclude that the improved growth performance of Nanfeng tangerine is either due to higher $\mathrm{pH}$ and organic matter or due to the fact that biochar and cake fertilizer provides nutrients more sustainably for plant growth. There was increased nutrient availability in the soil as a result of application of biochar and cake fertilizer, leading to increased uptake by Nanfeng tangerine.

The Nanfeng tangerine is seriously affected by inferior mastication, which is characterized by a coarse feeling in the mouth while eating with rough fiber residue after patiently chewing the fruit segments before swallowing (Lei et al., 2012). Mastication of fruit segment can be quantitatively evaluated using selected texture indices such as the shear force, the low shear force means superior mastication characteristic (Zheng et al., 2015). Thus, in our results, the decreases of shear forces as well as the fiber strength, ductility and tenacity implied an improved mastication characteristic by organic amendments such as biochar and cake fertilizer application. Additionally, the fruit size, peel coloration, and some key primary metabolites including sugars, organic acids and vitamin $\mathrm{C}$ were affected by the biochar application. Accumulation of soluble sugars and the decline in acid content result the low ratio of sugars to organic acids, making the fruit more suitable for taste and palatability. Citrus fruit quality is highly influenced by environmental factors and cultural practices, and complex interrelationships may exist among many of them to determine the final quality of the fruit (Zheng et al., 2015; Lado et al., 2018). So, the improvement of comprehensive quality in Nanfeng tangerine could be attributed to applications of biochar and cake fertilizer, which modify soil physicochemical parameters and affect plant nutritional status. Future studies may consider longterm field experiments to reveal the multiple effects of biochar application on soil and plants.

\section{Conclusion}

The present study revealed that application of biochar combined with cake fertilizer showed better results to improve the soil condition of citrus orchards than the solely application of biochar or cake fertilizer. The combined applications also improved leaf and shoot growth, increased leaf nutrient concentration, fruit mastication characteristics, fruit size, soluble sugars content, and decreased acid content of Nanfeng tangerine. Therefore, the combined application of biochar and cake fertilizer is a better measure to improve plant growth and fruit quality of citrus grown in acidic soils.

\section{Acknowledgments}

This work is funded by the National Natural Science Foundation of China (31260457), Key R\&D Initiative and Projects of Jiangxi Province (20171ACF60025), and Jiangxi Natural Science Foundation Project (20122BAB204013).

\section{References}

Abid, M., Danish, S., Zafar-Ul-Hye, M., Shaaban, M., and Naqqash, M.N. (2017). Biochar increased photosynthetic and accessory pigments in tomato (Solanum lycopersicum L.) plants by reducing cadmium concentration under various irrigation waters. Environ. Sci. Pollut. R. 24, 22111-22118. https://doi.org/10.1007/s11356-017-9866-8.

Abrishamkesh, S., Asadi, H., Bagheri-Marandi, G.H., and Pourbabaee, A.A. (2015). Effects of rice husk biochar application on the properties of alkaline soil and lentil growth. Plant Soil Environm. 61, 475-482. https://doi.org/10.17221/117/2015-PSE.

Adekiya, A.O., Agbede, T.M., Aboyeji, C.M., Dunsin, O., and Simeon, V.T. (2019). Effects of biochar and poultry manure on soil characteristics and the yield of radish. Sci. Hortic. (Amsterdam) 243, 1-13. https:// doi.org/10.1016/j.scienta.2018.08.048.

Alburquerque, J.A., Calero, J.M., Barrón, V., Torrent, J., del Campillo, M.C., Gallardo, A., and Villar, R. (2014). Effects of biochars produced from different feedstocks on soil properties and sunflower growth. J. Plant Nutr. Soil Sci. 177, 16-25. https://doi.org/10.1002/ jpln.201200652.

Al-Wabel, M.I., Hussain, Q., Usman, A.R.A., Ahmad, M., Abduljabbar, A., Sallam A.S., and Ok, Y.S. (2018). Impact of biochar properties on soil conditions and agricultural sustainability: A review. Land Degrad. Dev. 29, 2124-2161. https://doi.org/10.1002/ldr.2829.

Amendola, C., Montagnoli, A., Terzaghi, M., Trupiano, D., Oliva, F., Baronti, S., Migliettac, F., Chiatanteb, D., and Scippaa, G.S. (2017). Short-term effects of biochar on grapevine fine root dynamics and arbuscular mycorrhizae production. Agr. Ecosyst. Environm. 239, 236-245. https://doi.org/10.1016/j.agee.2017.01.025.

Arif, M., Ali, K., Jan, M.T., Shah, Z., and Quilliam, R.S. (2016). Integration of biochar with animal manure and nitrogen for improving maize yields and soil properties in calcareous semiarid agroecosystems. Field Crop Res. 195, 28-35. https://doi. org/10.1016/j.fcr.2016.05.011.

Bai, N., Zhang, H., Li, S., Zheng, X., Zhang, J., Zhang, H., Zhou, S., Sun, H., and Lv, W. (2019). Long-term effects of straw and strawderived biochar on soil aggregation and fungal community in a ricewheat rotation system. PeerJ. 6, e6171. https://doi.org/10.7717/ peerj.6171.

Chen, W., Meng, J., Han, X., Lan, Y., and Zhang, W. (2019). Past, present, and future of biochar. Biochar 1, 75-87. https://doi.org/10.1007/ s42773-019-00008-3.

Fischer, B.M.C., Manzoni, S., Morillas, L., Garcia, M., Johnson, M.S., and Lyon, S.W. (2019). Improving agricultural water use efficiency with biochar - A synthesis of biochar effects on water storage and fluxes across scales. Sci. Total Environm. 657, 853-862. https://doi. org/10.1016/j.scitotenv.2018.11.312.

Gao, J.F. (2006). Experimental Guidance for Plant Physiology. (Beijing: Higher Education Press).

Guo, C.X., Pan, Z.Y., and Peng, S.A. (2016). Effect of biochar on the growth of Poncirus trifoliata (L.) Raf. seedlings in Gannan acidic red soil. Soil Sci. Plant Nutr. 62, 194-200. https://doi.org/10.1080/003 80768.2016.1150789.

Joseph, U.E., Toluwase, A.O., Kehinde, E.O., Omasan, E.E., Yetunde, A.T., George, O.O., Zhao, C.S., and Wang, H.Y. (2020). Effect of biochar on soil structure and storage of soil organic carbon and nitrogen in the aggregate fractions of an albic soil. Arch. Agron. Soil Sci. 66, 1-12. https://doi.org/10.1080/03650340.2019.1587412. 
Khan, M., Fatima, K., Ahmad, R., Younas, R., Rizwan, M., Azam, M., Abadin, Z., and Ali, S. (2019a). Comparative effect of mesquite biochar, farmyard manure, and chemical fertilizers on soil fertility and growth of onion (Allium cepa L.). Arab. J. Geosci. 12, 653. https:// doi.org/10.1007/s12517-019-4734-0.

Khan, M.N., Lan, Z., Sial, T.A., Zhao, Y., Haseeb, A., Zhang, J.G., Zhang, A.F., and Hill, R.L. (2019b). Straw and biochar effects on soil properties and tomato seedling growth under different moisture levels. Arch. Agron. Soil Sci. 65, 1704-1719. https://doi.org/10.1080 /03650340.2019.1575510.

Lado, J., Gambetta, G., and Zacarias, L. (2018). Key determinants of citrus fruit quality: Metabolites and main changes during maturation. Sci. Hortic. (Amsterdam) 233, 238-248. https://doi.org/10.1016/j. scienta.2018.01.055.

Lei, Y., Liu, Y.Z., Gu, Q.Q., Yang, X.Y., Deng, X.X., and Chen, J.Y. (2012). Comparison of cell wall metabolism in the pulp of three cultivars of 'Nanfeng' tangerine differing in mastication trait. J. Sci. Food Agr. 92, 496-502. https://doi.org/10.1002/jsfa.4554.

Li, Z.G., Luo, Y.M., and Teng, Y. (2008). Methodology for Soil and Environmental Microorganism (Beijing: Science Press).

Liang, B., Lehmann, J., Solomon, D., Kinyangi, J., Grossman, J., O’Neill, B., Skjemstad, J.O., Thies, J.E., Luizão, F.J., Petersen, J., and Neves, E.G. (2006). Black carbon increases cation exchange capacity in soils. Soil Sci. Soc. Am. J. 70, 1719-1730. https://doi.org/10.2136/ sssaj2005.0383.

Liu, Y., Lu, H., Yang, S., and Wang, Y. (2016). Impacts of biochar addition on rice yield and soil properties in a cold waterlogged paddy for two crop seasons. Field Crop Res. 191, 161-167. https:// doi.org/10.1016/j.fcr.2016.03.003.

Major, J., Rondon, M., Molina, D., Riha, S.J., and Lehmann, J. (2010). Maize yield and nutrition during 4 years after biochar application to a Colombian savanna oxisol. Plant Soil 333, 117-128. https://doi. org/10.1007/s11104-010-0327-0.

Nguyen, T., Tran, N.N., Nguyen, T.T., Le, C.M.T., Tran, T.V., Thai, B.V., and Le, T.V. (2018). The interactive effects of biochar and cow manure on rice growth and selected properties of salt-affected soil. Arch. Agron. Soil Sci. 64, 1744-1758. https://doi.org/10.1080/03650340.2018.1 455186

Partey, S.T., Preziosi, R.F., and Robson, G.D. (2014). Short-term interactive effects of biochar, green manure, and inorganic fertilizer on soil properties and agronomic characteristics of Maize. Agri. Res. 3, 128-136. https://doi.org/10.1007/s40003-014-0102-1.

Pratiwi, E.P.A., and Shinogi, Y. (2016). Rice husk biochar application to paddy soil and its effects on soil physical properties, plant growth, and methane emission. Paddy Water Environm. 14, 521-532. https://doi.org/10.1007/s10333-015-0521-z.

Ramzani, P.M.A., Shan, L., Anjum, S., Khan, W.U.D., and Kausar, S. (2017). Improved quinoa growth, physiological response, and seed nutritional quality in three soils having different stresses by the application of acidified biochar and compost. Plant Physiol. Biochem. 116, 127-138. https://doi.org/10.1016/j.plaphy.2017.05.003.

Sandhu, S., Sekaran, U., Ozlu, E., Hoilett, N.O., and Kumar, S. (2019). Short-term impacts of biochar and manure application on soil labile carbon fractions, enzyme activity, and microbial community structure. Biochar 1, 271-282. https://doi.org/10.1007/s42773019-00025-2.

Schulz, H., and Glaser, B. (2012). Effects of biochar compared to organic and inorganic fertilizers on soil quality and plant growth in a greenhouse experiment. J. Plant Nutr. Soil Sci. 175, 410-422. https:// doi.org/10.1002/jpln.201100143.

Singh, R., Singh, P., Singh, H., and Raghubanshi, A.S. (2019). Impact of sole and combined application of biochar, organic and chemical fertilizers on wheat crop yield and water productivity in a dry tropical agro-ecosystem. Biochar 1, 229-235. https://doi.org/10.1007/ s42773-019-00013-6.

Steiner, C., Teixeira, W.G., Lehmann, J., Nehls, T., Macêdo, J.L.V., Blum, W.E.H., and Zech, W. (2007). Long term effects of manure, charcoal and mineral fertilization on crop production and fertility on a highly weathered central Amazonian upland soil. Plant Soil 291, 275-290. https://doi.org/10.1007/s11104-007-9193-9.

Uzoma, K.C., Inoue, M., Andry, H., Fujimaki, H., Zahoor, A., and Nishihara, E. (2011). Effect of cow manure biochar on maize productivity under sandy soil condition. Soil Use Manag. 27, 205212. https://doi.org/10.1111/j.1475-2743.2011.00340.x.

Wang, G., Govinden, R., Chenia, H.Y., Ma, Y., and Ren, G. (2019). Suppression of Phytophthora blight of pepper by biochar amendment is associated with improved soil bacterial properties. Biol. Fert. Soils 55, 813-824. https://doi.org/10.1007/s00374-019-01391-6.

Wang, Y., and Liu, R.H. (2017). Improvement of acidic soil properties by biochar from fast pyrolysis. Environ. Progr. Sustain. 37, 17431749. https://doi.org/10.1002/ep.12825.

Warnock, D.D., Lehmann, J., Kuyper, T.W., and Rillig, M.C. (2007). Mycorrhizal responses to biochar in soil - Concepts and mechanisms. Plant Soil 300, 9-20. https://doi.org/10.1007/s11104-007-9391-5.

Wei, Q.J., Wang, M.Q., Zeng, Z.F., Yang, C.Q., Peng, S.A., and Liu, Y.Z. (2014). Evaluation of the mastication and comparison of fruit quality with different bearing habits in Nanfeng tangerine (Citrus reticulata Blanco cv. Kinokuni). Sci. Agr. Sinica 47(6), 1162-1170.

Zhang, W.S., Chen, K.S., Zhang, B., Sun, C.D., Cai, C., Zhou, C.H., Xu, W.P., Zhang, W.Q., and Ferguson, I.B. (2005). Postharvest responses of Chinese bayberry fruit. Postharv. Biol. Technol. 37, 241-251. https:// doi.org/10.1016/j.postharvbio.2005.05.005.

Zhang, Y., Tan, Q.L., Hu, C.X., Zheng, C.S., Gui, H.P., Zeng, W.N., Sun, X.C., and Zhao, X.H. (2015). Differences in responses of soil microbial properties and trifoliate orange seedling to biochar derived from three feedstocks. J. Soil Sediment 15, 541-551. https://doi. org/10.1007/s11368-014-1032-z.

Zheng, C.S., Lan, X., Tan, Q.L., Zhang, Y., and Hu, C.X. (2015). Soil application of calcium and magnesium fertilizer influences the fruit pulp mastication characteristics of Nanfeng tangerine (Citrus reticulata Blanco cv. Kinokuni). Sci. Hortic. (Amsterdam) 191, 121126. https://doi.org/10.1016/j.scienta.2015.05.008.

Zwieten, L. van, Kimber, S., Morris, S., Chan, K.Y., Downie, A., Rust, J., Joseph, S., and Cowie, A. (2010). Effects of biochar from slow pyrolysis of papermill waste on agronomic performance and soil fertility. Plant Soil 327, 235-246. https://doi.org/10.1007/s11104009-0050-x.

Received: Feb. 18, 2020

Accepted: Jun. 17, 2020

Addresses of authors:

Qingjiang Wei ${ }^{1}$, Shouting $\mathrm{Su}^{1}$, Si Le ${ }^{1}$, Qiaoli $\mathrm{Ma}^{1}$, Xiao $\mathrm{Liu}^{2}$ and Qingqing $\mathrm{Gu}^{1, *}$

${ }^{1}$ College of Agronomy, Jiangxi Agricultural University,

Nanchang, Jiangxi, China

${ }^{2}$ School of Horticulture and Plant Protection, Yangzhou

University, Yangzhou, Jiangsu, China

* Corresponding author;

E-mail: qingqinggu2006@126.com 